\title{
CONTROLE DE Brevipalpus phoenicis (Geijskes, 1939) E Oligonychus ilicis (McGregor, 1917) (Acari: Tenuipalpidae, Tetranychidae) EM CAFEEIRO E O IMPACTO SOBRE ÁCAROS BENÉFICOS. I - ABAMECTIN E EMAMECTIN
}

\author{
Control of Brevipalpus phoenicis (Geijskes, 1939) and Oligonychus ilicis \\ (McGregor, 1917) (Acari: Tenuipalpidae, Tetranychidae) In coffee plants \\ and the impact on beneficial mites. I - Abamectin and emamectin
}

\author{
Paulo Rebelles Reis ${ }^{1}$, Marçal Pedro Neto ${ }^{2}$, Renato André Franco ${ }^{2}$, Adenir Vieira Teodoro ${ }^{2}$
}

\begin{abstract}
RESUMO
O ácaro Brevipalpus phoenicis (Geijskes, 1939) (Acari: Tenuipalpidae) é importante em cafeeiro (Coffea spp.), por ser o vetor do vírus da mancha-anular, responsável por queda de folhas e má qualidade da bebida do café, e o ácaro-vermelho, Oligonychus ilicis (McGregor, 1917) (Acari: Tetranychidae), por reduzir a área foliar de fotossíntese. Alguns ácaros da família Phytoseiidae são eficientes predadores associados aos ácaros-praga. Com este trabalho teve-se como objetivo estudar o controle dos ácaros-praga e o impacto do abamectin e emamectin sobre fitoseídeos. Em laboratório, foram estudados os efeitos ovicida, tópico, residual, tópico mais residual aos ácaros-praga e a seletividade fisiológica aos fitoseídeos. Em semicampo, foi estudada a persistência dos produtos no controle dos ácaros-praga. O efeito ovicida foi avaliado em ovos no início e fim de incubação; os efeitos residual, tópico e tópico mais residual foram avaliados pela mortalidade de larvas, ninfas e adultos após 48 horas da aplicação, enquanto a persistência foi avaliada pela mortalidade até 30 dias após a pulverização. A seletividade aos ácaros fitoseídeos foi avaliada pelo efeito total às fêmeas adultas, em teste residual em superfície de vidro. Pelos resultados, verificou-se que abamectin e emamectin não possuem ação ovicida, para ambas as espécies de ácaros-praga estudadas. Considerando o efeito tópico mais residual, o abamectin e emamectin foram altamente eficientes no controle de larvas, ninfas e adultos de $B$. phoenicis; apenas abamectin foi eficiente no controle de $O$. ilicis. Abamectin foi levemente a moderadamente nocivo e emamectin mostrou-se inócuo a levemente nocivo aos fitoseídeos. Devido à eficiência de controle e seletividade a fitoseídeos, conclui-se que abamectin e emamectin podem ser utilizados em programas de manejo integrado do ácaro B. phoenicis, e abamectin para o manejo de $B$. phoenicis e $O$. ilicis em cafeeiro.
\end{abstract}

Termos para indexação: Mancha-anular, controle químico, seletividade, ácaro, Coffea arabica.

\begin{abstract}
The mite Brevipalpus phoenicis (Geijskes, 1939) (Acari: Tenuipalpidae) is important for coffee plants (Coffea spp.) for being the vector of the coffee ringspot virus, which is responsible for leaf fall and bad quality of the coffee beverage. The red spider mite, Oligonychus ilicis (McGregor, 1917) (Acari: Tetranychidae) is also important, for reducing the areas of photosynthesis in leaves. Some mites of the family Phytoseiidae are efficient predators associated to the pest-mites. This work had as objective to study the control of the pest-mites and the impact of the abamectin and emamectin on phytoseiids. Studies were carried out in laboratory to investigate the ovicidal, topical, residual, topical plus residual effects of the products on the pest-mites and the physiologic selectivity to the phytoseiids. The persistence of the products in the control of pest-mite was studied in semi-field conditions. The ovicidal effect was evaluated in eggs in the beginning and end of incubation; the residual, topical and topical plus residual effects on larvae, nymphs and adults were assessed through mortality evaluation $48 \mathrm{~h}$ after spraying, while the persistence was evaluated until 30 days after spraying. The phytoseiids selectivity was evaluated by the total effect in adult females, in residual test in glass surface. The results showed that abamectin and emamectin do not possess ovicidal action, in both pest-mite species studied. Considering the topical plus residual effect, the abamectin and emamectin was highly efficient in the control of larvae, nymphs and adults of the B. phoenicis; only the abamectin was efficient for $O$. ilicis control. Abamectin was slightly and moderately noxious and emamectin was shown to be innocuous and slightly noxious
\end{abstract}

(Recebido para publicação em 16 de janeiro de 2003 e aprovado em 10 de junho de 2003)

\footnotetext{
1. Engenheiro Agrônomo, D.Sc., Pesquisador EPAMIG-CTSM/EcoCentro, bolsista do CNPq, Caixa Postal 176 - 37.200-000 - Lavras, MG. paulo.rebelles@epamig.ufla.br

2. Bolsistas de IC/CBP\&D-Café, estudantes de Agronomia da Universidade Federal de Lavras/UFLA - Caixa Postal 37 - $37200-000$ - Lavras, MG
} 
to the phytoseiids. Due to efficiency presented in the pest-mites control, and selectivity to the phytoseiids, it is concluded that abamectin and emamectin can be used in integrated pest management programs of B. phoenicis, and abamectin for the management of $B$. phoenicis and $O$. ilicis in coffee trees.

Index terms: Coffee ringspot virus, chemical control, selectivity, mites, Coffea arabica.

\section{INTRODUÇÃO}

O ácaro Brevipalpus phoenicis (Geijskes, 1939) (Acari: Tenuipalpidae) tem sido relatado em cafeeiros (Coffea spp.) no Brasil, pelo menos desde 1950 (A INFESTAÇÃO..., 1951; AMARAL, 1951), quando foi relatado no Estado de São Paulo como Tenuipalpus phoenicis, Geijskes, 1939, juntamente com surtos do ácaro-vermelho, Oligonychus ilicis (McGregor, 1917) (Acari: Tetranychidae), esse relatado como Paratetranychus ununguis Jacob, 1905 (CALZA e SAUER, 1952). Posteriormente, o ácaro B. phoenicis foi relacionado com a doença conhecida como mancha-anular do cafeeiro (CHAGAS, 1973), causada por um vírus do grupo dos Rhabdovirus (CHAGAS, 1988).

Até 1988, a mancha-anular do cafeeiro não tinha ainda representado problema econômico, embora, em 1986, tenha sido associada a uma intensa desfolha devido a um inverno com baixa precipitação pluvial, condição muito favorável ao ácaro (CHAGAS, 1988).

Desde 1990, com destaque para 1995, a infestação de $B$. phoenicis e a incidência da mancha-anular têm sido relatadas em Minas Gerais como causa de intensa desfolha em cafeeiros, principalmente na região do Alto Paranaíba (FIGUEIRA et al., 1996). Também foi constatada a presença do ácaro nas demais regiões cafeeiras do Brasil, tanto em cafeeiro arábica (Coffea arabica, L.), quanto canéfora (Coffea canephora Pierre \& Froenher) (MATIELLO, 1987).

B. phoenicis é cosmopolita, infestando diversas espécies vegetais. No Brasil, Reis (1974) citou 37 hospedeiros do ácaro, principalmente em fruteiras, e Trindade e Chiavegato (1994) citaram 33 hospedeiros, principalmente plantas invasoras e ornamentais.

B. phoenicis, conhecido como ácaro-plano ou da leprose, é uma séria praga da cultura dos citros (CHIAVEGATO et al., 1982; CHIAVEGATO, 1991), pois ataca as folhas, ramos e principalmente os frutos (CHIAVEGATO e KHARFAN, 1993), causando prejuízos. Seu controle em citros tem sido realizado a cada ano.

B. phoenicis é importante em cafeeiro por ser o vetor do vírus da mancha-anular, responsável por queda de folhas e má qualidade da bebida do café (REIS e CHAGAS, 2001).

O ácaro-vermelho, O. ilicis, já foi referido como a segunda praga em importância para o cafeeiro conilon
(C. canephora) no Estado do Espírito Santo (IBC, 1985), que é considerado mais sensível ao ácaro do que o arábica (C. arabica). Para se alimentar, principalmente na página superior das folhas, perfura as células e absorve parte do conteúdo celular. Em conseqüência, as folhas perdem o brilho natural, tornam-se bronzeadas, dando um péssimo aspecto às plantas. $\mathrm{O}$ ataque ocorre geralmente em reboleiras, porém, se as condições forem favoráveis ao ácaro e o controle não for feito no início da infestação, poderá atingir toda a lavoura. Períodos de seca, com estiagem prolongada, são condições propícias à proliferação do ácaro, podendo causar desfolha das plantas, e lavouras em formação podem ter seu desenvolvimento retardado (REIS e SOUZA, 1986).

Ácaros da família Phytoseiidae estão associados a ácaros do cafeeiro (PALLINI FILHO et al., 1992; REIS et al., 2000) e são os mais importantes e estudados predadores de ácaros-praga (MCMURTRY e CROFT, 1997).

O controle químico de ácaros em cafeeiros no Brasil, principalmente de B. phoenicis, é muito pouco conhecido, ao contrário do que ocorre em citros. Alguns produtos como cyhexatin, azocyclotin (PM), bromopropilato, fenpyroximate e meothrin mostraram ser eficientes no controle de B. phoenicis em cafeeiro (PAPA, 1997; OLIVEIRA e REIFF, 1998), porém, esses pesquisadores não relataram a seletividade aos predadores. Reis et al. (2002) destacaram como eficientes no controle de $B$. phoenicis, e seletivos aos fitoseídeos, o hexythiazox, fenbutatin oxide, enxofre e abamectin.

Considerando os aspectos mencionados, objetivou-se especificamente com este ensaio avaliar o efeito dos produtos abamectin (Vertimec $18 \mathrm{CE}$ ) e emamectin (Proclaim $5 \mathrm{SG}$ ) sobre os ácarospraga do cafeeiro e a seletividade a ácaros predadores pertencentes à família Phytoseiidae a eles associados.

\section{MATERIAL E MÉTODOS}

Os experimentos foram realizados no Centro de Pesquisa em Manejo Ecológico de Pragas e Doenças de Plantas - EcoCentro, da Empresa de Pesquisa Agropecuária de Minas Gerais - EPAMIG, no campus da UFLA. À exceção do estudo da persistência em casade-vegetação, os demais experimentos foram feitos Ciênc. agrotec., Lavras, v. 28, n. 2, p. 269-281, mar./abr., 2004 
em laboratório a $25 \pm 2{ }^{\circ} \mathrm{C}, 70 \pm 10 \%$ de UR e 14 horas de fotofase.

\section{Criação de manutenção}

A criação de manutenção do ácaro-praga B. phoenicis, para uso nos bioensaios, foi feita em folhas de cafeeiro, à semelhança das arenas utilizadas nos testes com produtos fitossanitários, ou em laranjas parafinadas, conforme metodologia descrita por Chiavegato (1986). A criação de $O$.ilicis foi feita em folhas de cafeeiro conforme metodologia descrita por Reis et al. (1997). Os ácaros Phytoseiidae utilizados nos testes de seletividade foram oriundos de criação de manutenção em laboratório, realizada conforme metodologia descrita por Reis e Alves (1997).

\section{Procedimentos Experimentais no Laboratório}

Os testes foram realizados em folhas de cafeeiro (C. arabica) coletadas de plantas sem tratamento fitossanitário, que serviram de fonte de alimento aos ácaros e de arena. As folhas foram colocadas sobre uma esponja de $2 \mathrm{~cm}$ de espessura, constantemente umedecida com água destilada, que ocupava todo o interior de uma placa de Petri de $15 \mathrm{~cm}$ de diâmetro por $2 \mathrm{~cm}$ de profundidade, sem tampa. Uma fina camada de algodão hidrófilo, de aproximadamente $2 \mathrm{~cm}$ de largura, foi colocada recobrindo todo o bordo da folha e ficando em contato com a espuma umedecida. A folha foi dividida em três partes por duas tiras de algodão, constituindo-se, assim, em três arenas. A água, além de manter a turgescência da folha, serviu de barreira, mantendo os ácaros sobre as arenas (REIS et al., 1997). Os produtos estudados e dosagens para 1000 litros de água são relatados na Tabela 1. A aplicação foi feita em torre de Potter a uma pressão de $15 \mathrm{lb} / \mathrm{pol}^{2}$, com a mesa de pulverização a uma distância de $1,7 \mathrm{~cm}$ do tubo de pulverização. Exceto no estudo do efeito tópico onde somente os ácaros foram pulverizados, cada folha recebeu um depósito de calda da ordem de $1,68 \pm 0,36 \mathrm{mg} / \mathrm{cm}^{2}$, em conformi- dade com o proposto pela IOBC/WPRS (HASSAN et al., 1994), representando o que ocorre quando é feita uma aplicação em campo. Para cada tratamento, foram utilizadas seis repetições.

\section{Efeito ovicida}

Fêmeas adultas dos ácaros foram confinadas nas arenas durante dois dias para a obtenção de ovos, dos quais foram aproveitados 20 ovos/arena. Os ovos assim obtidos foram pulverizados com os produtos em teste e observados diariamente até a eclosão das larvas. Foram feitos testes com ovos de B. phoenicis com dois e oito dias de incubação, ou seja, ovos no início e no final do período de incubação (CHIAVEGATO, 1986) e ovos de $O$. ilicis com dois e seis dias de incubação, também no início e no fim do período de incubação (REIS et al., 1997). Quando houve eclosão de larva, o produto foi considerado sem efeito ovicida.

\section{Efeito tópico}

Foi estudado o efeito dos produtos quando aplicados diretamente sobre o idiossoma do ácaro. Após a pulverização, dez ácaros de cada fase pós-embrionária (larva, ninfa e adulto) foram colocados em arenas nãopulverizadas, em experimentos separados para cada fase e espécie, avaliando-se a mortalidade 48 horas mais tarde.

\section{Efeito residual}

Neste experimento, foi estudado o efeito residual dos produtos sobre as folhas na mortalidade do ácaro. Depois da pulverização, as folhas foram secas em condições ambientes do laboratório por 1 hora. Em seguida, foram colocados dez ácaros de cada fase pósembrionária em cada uma das três partes em que foi dividida cada folha, em experimentos separados para cada fase e espécie, avaliando-se a mortalidade 48 horas mais tarde. 
TABELA 1 - Número médio de larvas de Brevipalpus phoenicis eclodidas de ovos pulverizados após dois e oito dias de incubação e eficiência ovicida (\% Efic.) dos produtos testados $(n=20)$.

\begin{tabular}{|c|c|c|c|c|c|}
\hline \multirow{2}{*}{ Tratamentos } & \multirow{2}{*}{$\begin{array}{c}\text { Dosagem } \\
\text { por ha }^{1}\end{array}$} & \multicolumn{2}{|c|}{ Dois dias } & \multicolumn{2}{|c|}{ Oito dias } \\
\hline & & Média $^{2}$ & Efic. $(\%)$ & Média $^{2}$ & Efic. $(\%)$ \\
\hline Testemunha & - & $19,3 \mathrm{a}$ & - & $19,2 \mathrm{a}$ & - \\
\hline Abamectin (Vertimec $18 \mathrm{CE}$ ) & 300 & $18,2 \mathrm{a}$ & 5,7 & $18,3 \mathrm{a}$ & 4,7 \\
\hline Emamectin (Proclaim 5 SG) & 200 & $15,0 \mathrm{a}$ & 22,3 & $17,0 \mathrm{a}$ & 11,5 \\
\hline Emamectin (Proclaim 5 SG) & 400 & $15,0 \mathrm{a}$ & 22,3 & $14,3 \mathrm{a}$ & 25,5 \\
\hline Fenbutatin oxide (Torque $500 \mathrm{SC}$ ) & 800 & $8,5 \mathrm{~b}$ & 56,0 & $8,5 \mathrm{~b}$ & 55,7 \\
\hline Azocyclotin (Peropal 250 PM) ${ }^{3}$ & 1000 & $8,5 \mathrm{~b}$ & 56,0 & $7,3 \mathrm{~b}$ & 62,0 \\
\hline Hexythiazox (Savey $500 \mathrm{PM})^{3}$ & 30 & $1,5 \mathrm{c}$ & 92,2 & $6,5 \mathrm{~b}$ & 66,1 \\
\hline $\mathrm{CV}(\%)$ & & 13,7 & & 14,3 & \\
\hline
\end{tabular}

${ }^{1}$ Dosagem em g ou ml / ha ou 1000 litros de água.

${ }^{2}$ Médias seguidas de mesma letra na coluna não diferem entre si pelo teste de Duncan $(\mathbf{p}<0,05)$.

${ }^{3}$ Padrões de controle neste experimento.

\section{Efeito tópico mais residual}

Neste experimento, foram estudados os dois efeitos em conjunto. Dez ácaros de cada fase pósembrionária, em experimentos separados para cada fase e espécie, foram colocados nas arenas antes da pulverização. Os ácaros receberam o produto sobre o idiossoma e permaneceram em contato com o resíduo do mesmo sobre a folha, avaliando-se a mortalidade 48 horas mais tarde.

\section{Semicampo}

Nesta condição, foi avaliada a persistência de cada produto no controle do ácaro da mancha-anular e vermelho do cafeeiro. Foram utilizadas mudas de cafeeiro (C. arabica) de aproximadamente $1 \mathrm{~m}$ de altura, com 30 a 40 folhas, plantadas em vasos plásticos de 10 litros, em casa-de-vegetação. Essas foram pulverizadas uma única vez com os mesmos produtos e sob as mesmas dosagens citadas no bioensaio, para verificar o efeito ovicida (Tabela 1). Os produtos foram aplicados até o ponto de escorrimento, utilizando-se um pulverizador de pressão constante com capacidade de 1 litro. Após a aplicação de cada produto, o pulverizador foi lavado com água corrente, e depois com álcool absoluto, a fim de evitar o efeito de resíduos do produto utilizado sobre outros produtos a serem aplicados posteriormente.
Os bioensaios para verificar a persistência dos acaricidas foram realizados aos $0,5,15$ e 30 dias após a aplicação (DAA) em folhas destacadas das plantas tratadas, sendo os vasos mantidos na casa-devegetação. Foram confeccionadas no laboratório, com as folhas coletadas, arenas de $3 \mathrm{~cm}$ de diâmetro. As arenas foram colocadas para flutuar em água em uma placas de Petri de $15 \mathrm{~cm}$ de diâmetro x $2 \mathrm{~cm}$ de profundidade, sem tampa, conforme metodologia descrita por Reis et al. (1998). Para cada tratamento, foram utilizadas seis repetições. Em cada arena, foram colocados dez ácaros de uma das fases a ser avaliada (larva, ninfa e adulta), oriundos da criação de manutenção. Após 48 horas foram contados os ácaros sobreviventes.

Após os testes, cada produto foi classificado em uma das quatro classes de persistência (HASSAN et al., 1994), de acordo com o período em que pelo menos $80 \%$ dos ácaros morreram: classe 1 - baixa persistência (até 5 dias); classe 2 - levemente persistente (6 a 15 dias); classe 3 - moderadamente persistente (16 a 30 dias) e classe 4 - persistentes (mais de 30 dias).

\section{Seletividade a ácaros predadores}

Foi estudada sobre duas espécies de ácaros da família Phytoseiidae comumente associadas aos ácarospraga do cafeeiro, Euseius alatus DeLeon e Euseius citri- 
folius Denmark \& Muma (PALLINI et al., 1992; REIS et al., 2000). Os ácaros fitoseídeos utilizados nos testes foram oriundos de criação de manutenção em laboratório. Foi utilizado o método residual da pulverização em superfície de vidro, recomendado como padrão para testes em laboratório de efeitos adversos de produtos fitossanitários a ácaros predadores pela IOBC / WPRS ("International Organization for Biological and Integrated Control of Noxious Animals and Plants / West Paleartic Regional Section") (HASSAN et al., 1994). Lamínulas de vidro de 20x20 mm, flutuando em água numa placa de Petri de 5 $\mathrm{cm}$ de diâmetro x $2 \mathrm{~cm}$ de profundidade, sem tampa, foram usadas como superfície para aplicação dos produtos, e suporte para os ácaros (REIS et al., 1998, 1999). O efeito residual foi medido por meio do efeito adverso ou total (E\%) calculado, levando em conta a mortalidade no tratamento (corrigida em função da mortalidade na testemunha) e o efeito na reprodução (OVERMEER e ZON, $1982)$, sendo $E \%=100 \%-\left(100 \%-M_{c}\right) \times E_{r}$, em que $\mathrm{M}_{\mathrm{c}}=$ mortalidade corrigida (ABBOTT, 1925) e $\mathrm{E}_{\mathrm{r}}=\mathrm{e}-$ feito na reprodução. Durante oito dias, foram diariamente contadas as fêmeas vivas, e retiradas as mortas, bem como o número de ovos viáveis postos (que deram origem a larvas). $\mathrm{O}$ efeito na reprodução $\left(\mathrm{E}_{\mathrm{r}}\right)$ foi obtido pela divisão da produção média de ovos nos tratamentos pela produção média de ovos na testemunha $\left(\mathrm{E}_{\mathrm{r}}=\mathrm{R}_{\text {trata- }}\right.$ mento $\left./ \mathrm{R}_{\text {testemunha }}\right)$. A produção média de ovos $(\mathrm{R})$ foi obtida por meio da relação: $\mathrm{R}=$ número de ovos viáveis /número de fêmeas vivas. Foram considerados como válidos somente os testes em que a mortalidade na testemunha foi no máximo em torno de $20 \%$ (BAKKER et al., 1992). Os valores dos efeitos totais, encontrados para cada produto e dosagem testados, foram classificados nas classes 1 a 4, conforme critérios estabelecidos pela IOBC / WPRS (BAKKER et al., 1992; HASSAN et al., 1994): classe $1=$ $\mathrm{E}<30 \%$ (inócuo ou não nocivo); classe $2=30 \%<\mathrm{E}<$ $79 \%$ (levemente nocivo); classe $3=80 \%<\mathrm{E}<99 \%$ (moderadamente nocivo) e classe $4=\mathrm{E}>99 \%$ (nocivo).

\section{RESULTADOS E DISCUSSÃO}

\section{Efeito ovicida}

$\mathrm{O}$ acaricida hexythiazox (padrão como ovicida neste trabalho) mostrou-se um eficiente ovicida para $B$. phoenicis, principalmente quando aplicado sobre ovos com dois dias de incubação, com eficiência acima de $92 \%$. Porém, para aplicação sobre ovos mais velhos, ou seja, com maior tempo de incubação (oito dias), a eficiência ovicida foi reduzida para cerca de $66 \%$. O fenbutatin oxide e azocyclotin (padrões como controle de fases pósembrio-nárias) alcançaram cerca de $60 \%$ de eficiência como ovicida, para ovos com dois ou oito dias de incubação. $\mathrm{O}$ abamectin e emamectin foram pouco eficientes como ovicidas a B. phoenicis (Tabela 1). O ótimo efeito ovicida de hexythiazox, e parcial do fenbutatin oxide, para B. phoenicis, também foram demonstrados por Reis et al. (2002).

Para $O$. ilicis, somente o azocyclotin apresentou efeito ovicida, com $75 \%$ de eficiência em ovos com até dois dias de incubação, eficiência que foi reduzida para $44 \%$ em ovos com seis dias de incubação. O fenbutatin oxide apresentou $25 \%$ e $21,5 \%$ de eficiência ovicida para $O$. ilicis com dois e seis dias de incubação, respectivamente. O hexythiazox, padrão ovicida para $B$. phoenicis, não apresentou efeito ovicida sobre $O$. ilicis, nos dois tempos de incubação estudados. O efeito ovicida diminuiu com a idade do ovo. Abamectin e emamectin também não apresentaram efeito ovicida para $O$. ilicis (Tabela 2).

\section{Efeito tópico}

Quando os produtos foram aplicados sobre os ácaros, o abamectin, o fenbutatin oxide e o azocyclotin apresentaram eficiências acima de $80 \%$ no controle de todas as fases pós-embrionárias de B. phoenicis. Já emamectin, na dosagem de $400 \mathrm{~g} / \mathrm{ha}$, foi eficiente apenas no controle das larvas e ninfas (eficiência acima de $80 \%$ ) e apresentou cerca de $60 \%$ de eficiência no controle de adultos. O emamectin na menor dosagem $(200 \mathrm{~g} / \mathrm{ha})$ e o hexythiazox não apresentaram eficiência no controle do ácaro (Tabela 3). Para O. ilicis, somente o abamectin e fenbutatin oxide apresentaram eficiência de controle acima de $80 \%$ para as larvas, ninfas e adultos (Tabela 4).

\section{Efeito residual}

Abamectin, emamectin (nas duas dosagens testadas), fenbutatin oxide e azocyclotin foram altamente eficientes no controle de todas as fases pós-embrionárias de B. phoenicis. O hexythiazox não apresentou eficiência de controle para nenhuma fase pós-embrionária de B. phoenicis (Tabela 5). Para O. ilicis, abamectin, fenbutatin oxide e azocyclotin foram altamente eficientes no controle de todas as fases pós-embrionárias. $\mathrm{O}$ emamectin apresentou eficiência no controle de larvas de $O$. ilicis apenas na maior dosagem estudada (400 g/ha). O hexythiazox não apresentou eficiência no controle de nenhuma fase pósembrionária de $O$. ilicis (Tabela 6). 
TABELA 2 - Número médio de larvas de Oligonychus ilicis eclodidas de ovos pulverizados após dois e seis dias de incubação e eficiência ovicida (\% Efic.) dos produtos testados $(\mathrm{n}=20)$.

\begin{tabular}{|c|c|c|c|c|c|}
\hline \multirow{2}{*}{ Tratamentos } & \multirow{2}{*}{$\begin{array}{c}\text { Dosagem } \\
\text { por ha }^{1}\end{array}$} & \multicolumn{2}{|c|}{ Dois dias } & \multicolumn{2}{|c|}{ Seis dias } \\
\hline & & Média $^{2}$ & Efic. $(\%)$ & Média $^{2}$ & \% Efic. \\
\hline Testemunha & - & $20,0 \mathrm{a}$ & - & $20,0 \mathrm{a}$ & - \\
\hline Abamectin (Vertimec $18 \mathrm{CE})$ & 300 & $20,0 \mathrm{a}$ & 0,0 & $19,5 \mathrm{ab}$ & 2,5 \\
\hline Emamectin (Proclaim 5 SG) & 200 & $19,7 \mathrm{a}$ & 1,5 & $19,5 \mathrm{ab}$ & 2,5 \\
\hline Emamectin (Proclaim $5 \mathrm{SG}$ ) & 400 & $18,8 \mathrm{a}$ & 6,0 & $19,8 \mathrm{a}$ & 1,0 \\
\hline Fenbutatin oxide (Torque $500 \mathrm{SC})^{3}$ & 800 & $15,0 \mathrm{a}$ & 25,0 & $15,7 \mathrm{~b}$ & 21,5 \\
\hline Azocyclotin (Peropal 250 PM) ${ }^{3}$ & 1000 & $5,0 \mathrm{~b}$ & 75,0 & $11,2 \mathrm{c}$ & 44,0 \\
\hline Hexythiazox (Savey $500 \mathrm{PM})^{3}$ & 30 & $18,7 \mathrm{a}$ & 6,5 & $19,5 \mathrm{ab}$ & 2,5 \\
\hline $\mathrm{CV}(\%)$ & & 11,40 & & 8,7 & \\
\hline
\end{tabular}

${ }^{1}$ Dosagem em g ou ml / ha ou 1000 litros de água.

${ }^{2}$ Médias seguidas de mesma letra na coluna não diferem entre si pelo teste de Duncan $(\mathbf{p}<0,05)$.

${ }^{3}$ Padrões de controle neste experimento.

TABELA 3 - Número médio de larvas, ninfas e adultos de Brevipalpus phoenicis vivos 48 horas após a aplicação tópica dos tratamentos e porcentagem de eficiência de controle (\% Efic.) $(\mathrm{n}=10)$.

\begin{tabular}{|c|c|c|c|c|c|c|c|}
\hline \multirow[b]{2}{*}{ Tratamentos } & \multirow{2}{*}{$\begin{array}{c}\text { Dosagem } \\
\text { por ha }\end{array}$} & \multicolumn{2}{|c|}{ Larvas } & \multicolumn{2}{|c|}{ Ninfas } & \multicolumn{2}{|c|}{ Adultos } \\
\hline & & Média $^{2}$ & $\begin{array}{c}\% \\
\text { Efic. }\end{array}$ & Média $^{2}$ & $\begin{array}{c}\% \\
\text { Efic. }\end{array}$ & Média $^{2}$ & $\begin{array}{c}\% \\
\text { Efic. }\end{array}$ \\
\hline Testemunha & - & $9,8 \mathrm{a}$ & - & $10,0 \mathrm{a}$ & - & $9,7 \mathrm{a}$ & - \\
\hline Abamectin (Vertimec 18 CE) & 300 & $0,3 \mathrm{~d}$ & 96,9 & $0,5 \mathrm{~d}$ & 95,0 & $0,5 \mathrm{c}$ & 94,8 \\
\hline Emamectin (Proclaim 5 SG) & 200 & $3,3 \mathrm{~b}$ & 66,3 & $7,0 \mathrm{~b}$ & 30,0 & $3,7 \mathrm{~b}$ & 61,8 \\
\hline Emamectin (Proclaim 5 SG) & 400 & $0,0 \mathrm{~d}$ & 100,0 & $1,7 \mathrm{c}$ & 83,0 & $4,0 \mathrm{~b}$ & 58,8 \\
\hline Fenbutatin oxide (Torque 500 SC) & 800 & $1,7 \mathrm{c}$ & 82,7 & $0,5 \mathrm{~d}$ & 95,0 & $0,8 \mathrm{c}$ & 91,8 \\
\hline Azocyclotin (Peropal 250 PM) & 1000 & $0,0 \mathrm{~d}$ & 100,0 & $0,0 \quad \mathrm{~d}$ & 100,0 & $1,3 \mathrm{c}$ & 86,6 \\
\hline Hexythiazox (Savey 500 PM) & 30 & $8,3 \mathrm{a}$ & 15,3 & $9,5 \mathrm{a}$ & 5,0 & $10,0 \mathrm{a}$ & 0,0 \\
\hline $\mathrm{CV}(\%)$ & & 15,0 & & 18,3 & & 18,4 & \\
\hline
\end{tabular}

${ }^{1} \mathrm{~g}$ ou ml do produto comercial / 1000 litros de água.

${ }^{2}$ Médias seguidas de mesma letra na coluna não diferem entre si pelo teste de Duncan $(\mathbf{p}<0,05)$. 
TABELA 4 - Número médio de larvas, ninfas e adultos de Oligonychus ilicis vivos 48 horas após a aplicação tópica dos tratamentos e porcentagem de eficiência de controle (\% Efic.) $(\mathrm{n}=10)$.

\begin{tabular}{|c|c|c|c|c|c|c|c|}
\hline \multirow[b]{2}{*}{ Tratamentos } & \multirow{2}{*}{$\begin{array}{c}\text { Dosagem } \\
\text { por ha }^{1}\end{array}$} & \multicolumn{2}{|c|}{ Larvas } & \multicolumn{2}{|c|}{ Ninfas } & \multicolumn{2}{|c|}{ Adultos } \\
\hline & & Média $^{2}$ & $\begin{array}{c}\% \\
\text { Efic. }\end{array}$ & Média $^{2}$ & $\begin{array}{c}\% \\
\text { Efic. }\end{array}$ & Média $^{2}$ & $\begin{array}{c}\% \\
\text { Efic. }\end{array}$ \\
\hline Testemunha & - & $10,0 \mathrm{a}$ & - & $10,0 \mathrm{a}$ & - & $9,7 \mathrm{a}$ & - \\
\hline Abamectin (Vertimec 18 CE) & 300 & $0,0 \quad \mathrm{e}$ & 100,0 & $0,0 \mathrm{~d}$ & 100,0 & $0,5 \mathrm{~d}$ & 95,0 \\
\hline Emamectin (Proclaim 5 SG) & 200 & $9,8 \mathrm{a}$ & 2,0 & $6,8 \mathrm{~b}$ & 32,0 & $6,8 \mathrm{~b}$ & 30,0 \\
\hline Emamectin (Proclaim 5 SG) & 400 & $4,5 \mathrm{c}$ & 55,0 & $6,0 \mathrm{~b}$ & 40,0 & $3,8 \mathrm{c}$ & 61,0 \\
\hline Fenbutatin oxide (Torque $500 \mathrm{SC}$ ) & 800 & $1,0 \quad \mathrm{~d}$ & 90,0 & $0,8 \mathrm{c}$ & 92,0 & $0,7 \mathrm{~d}$ & 93,0 \\
\hline Azocyclotin (Peropal 250 PM) & 1000 & $6,2 \mathrm{~b}$ & 38,0 & $6,5 \mathrm{~b}$ & 35,0 & $6,0 \mathrm{~b}$ & 38,0 \\
\hline Hexythiazox (Savey 500 PM) & 30 & $8,7 \mathrm{a}$ & 13,0 & $8,8 \mathrm{a}$ & 12,0 & $9,5 \mathrm{a}$ & 2,0 \\
\hline $\mathrm{CV}(\%)$ & & 10,6 & & 9,8 & & 13,2 & \\
\hline
\end{tabular}

1 g ou ml do produto comercial / 1000 litros de água.

${ }^{2}$ Médias seguidas de mesma letra na coluna não diferem entre si pelo teste de Duncan $(\mathbf{p}<0,05)$.

TABELA 5 - Número médio de larvas, ninfas e adultos de Brevipalpus phoenicis vivos em função do efeito residual (resíduo fresco e seco logo após a aplicação) dos tratamentos e porcentagem de eficiência de controle (\% Efic.) (n $=10$ ).

\begin{tabular}{|c|c|c|c|c|c|c|c|}
\hline \multirow{2}{*}{ Tratamentos } & \multirow{2}{*}{$\begin{array}{c}\text { Dosagem } \\
\text { por ha }\end{array}$} & \multicolumn{2}{|c|}{ Larvas } & \multicolumn{2}{|c|}{ Ninfas } & \multicolumn{2}{|c|}{ Adultos } \\
\hline & & Média $^{2}$ & $\begin{array}{c}\% \\
\text { Efic. }\end{array}$ & Média $^{2}$ & $\begin{array}{c}\% \\
\text { Efic. }\end{array}$ & Média $^{2}$ & $\begin{array}{c}\% \text { E- } \\
\text { fic. }\end{array}$ \\
\hline Testemunha & - & $10,0 \mathrm{a}$ & - & $10,0 \mathrm{a}$ & - & $10,0 \mathrm{a}$ & - \\
\hline Abamectin (Vertimec $18 \mathrm{CE}$ ) & 300 & $0,0 \mathrm{~b}$ & 100,0 & $0,0 \mathrm{c}$ & 100,0 & $0,0 \mathrm{c}$ & 100,0 \\
\hline Emamectin (Proclaim 5 SG) & 200 & $0,0 \mathrm{~b}$ & 100,0 & $0,0 \mathrm{c}$ & 100,0 & $0,0 \mathrm{c}$ & 100,0 \\
\hline Emamectin (Proclaim 5 SG) & 400 & $0,0 \mathrm{~b}$ & 100,0 & $0,0 \mathrm{c}$ & 100,0 & $0,0 \mathrm{c}$ & 100,0 \\
\hline Fenbutatin oxide (Torque $500 \mathrm{SC}$ ) & 800 & $0,0 \mathrm{~b}$ & 100,0 & $0,0 \mathrm{c}$ & 100,0 & $0,0 \mathrm{c}$ & 100,0 \\
\hline Azocyclotin (Peropal 250 PM) & 1000 & $0,0 \mathrm{~b}$ & 100,0 & $0,0 \mathrm{c}$ & 100,0 & $0,0 \mathrm{c}$ & 100,0 \\
\hline Hexythiazox (Savey 500 PM) & 30 & $10,0 \mathrm{a}$ & 0,0 & $5,0 \mathrm{~b}$ & 50,0 & $7,7 \mathrm{~b}$ & 23,0 \\
\hline $\mathrm{CV}(\%)$ & & 1,7 & & 42,0 & & 7,1 & \\
\hline
\end{tabular}

${ }^{1}$ g ou ml do produto comercial / 1000 litros de água.

${ }^{2}$ Médias seguidas de mesma letra na coluna não diferem entre si pelo teste de Duncan $(\mathbf{p}<0,05)$. 
TABELA 6 - Número médio de larvas, ninfas e adultos de Oligonychus ilicis vivos em função do efeito residual (resíduo fresco e seco logo após a aplicação) dos tratamentos e porcentagem de eficiência de controle (\% Efic.) $(\mathrm{n}=10)$.

\begin{tabular}{|c|c|c|c|c|c|c|c|}
\hline \multirow[b]{2}{*}{ Tratamentos } & \multirow{2}{*}{$\begin{array}{c}\text { Dosagem } \\
\text { por ha }^{1}\end{array}$} & \multicolumn{2}{|c|}{ Larvas } & \multicolumn{2}{|c|}{ Ninfas } & \multicolumn{2}{|c|}{ Adultos } \\
\hline & & Média $^{2}$ & $\begin{array}{c}\% \text { E- } \\
\text { fic. }\end{array}$ & Média $^{2}$ & $\begin{array}{c}\% \\
\text { Efic. }\end{array}$ & Média $^{2}$ & $\begin{array}{c}\% \\
\text { Efic. }\end{array}$ \\
\hline Testemunha & - & $10,0 \mathrm{a}$ & - & $9,8 \mathrm{a}$ & - & $9,8 \mathrm{a}$ & - \\
\hline Abamectin (Vertimec $18 \mathrm{CE})$ & 300 & $0,0 \quad \mathrm{~d}$ & 100,0 & $0,0 \quad \mathrm{e}$ & 100,0 & $0,0 \mathrm{c}$ & 100,0 \\
\hline Emamectin (Proclaim 5 SG) & 200 & $5,3 \mathrm{~b}$ & 47,0 & $7,2 \mathrm{~b}$ & 26,5 & 8,7 a & 11,2 \\
\hline Emamectin (Proclaim 5 SG) & 400 & $1,2 \mathrm{c}$ & 88,0 & $4,2 \mathrm{c}$ & 57,0 & $6,7 \mathrm{~b}$ & 31,6 \\
\hline Fenbutatin oxide (Torque 500 SC) & 800 & $0,0 \quad \mathrm{~d}$ & 100,0 & $1,2 \mathrm{~d}$ & 87,8 & $0,7 \mathrm{c}$ & 92,9 \\
\hline Azocyclotin (Peropal 250 PM) & 1000 & $0,0 \quad \mathrm{~d}$ & 100,0 & $0,0 \quad \mathrm{e}$ & 100,0 & $0,0 \mathrm{c}$ & 100,0 \\
\hline Hexythiazox (Savey 500 PM) & 30 & $9,8 \mathrm{a}$ & 2,0 & $9,8 \mathrm{a}$ & 0,0 & $9,5 \mathrm{a}$ & 3,1 \\
\hline $\mathrm{CV}(\%)$ & & 18,5 & & 13,9 & & 12,8 & \\
\hline
\end{tabular}

1 $\mathrm{g}$ ou ml do produto comercial / 1000 litros de água.

${ }^{2}$ Médias seguidas de mesma letra na coluna não diferem entre si pelo teste de Duncan $(\mathbf{p}<0,05)$.

\section{Efeito tópico mais residual}

O efeito da aplicação tópica mais o efeito residual proporcionou aos produtos e dosagens estudadas eficiências acima de $80 \%$ de controle para todas as fases pósembrionárias de $B$. phoenicis, com exceção do hexythiazox, que não controlou eficientemente nenhuma fase pós-embrionária do ácaro (Tabela 7). Para O. ilicis, o abamectin e fenbutatin oxide apresentaram o mesmo padrão de controle observado para o efeito tópico, porém, o azocyclotin, que não apresentou controle só com aplicação tópica, apresentou alta eficiência de controle quando somados os efeitos tópico mais residual, para todas as fases pós-embrionárias do ciclo do desenvolvimento de $O$. ilicis. Emamectin apresentou melhor efeito na maior dosagem (400 g/ha), com $67 \%, 57 \%$ e $54 \%$ de eficiência no controle de larvas, ninfas e adultos, respectivamente. $\mathrm{O}$ hexythiazox não controlou nenhuma fase pósembrionária (Tabela 8). Boa eficiência do abamectin e emamectin na redução da população das duas espécies de ácaros-praga em cafeeiro em condições de campo foi comprovada por Bevenga et al. (2002) e do abamectin na redução de B. phoenicis, por Reis et al. (2002).

A análise dos resultados, para ambas as espécies de ácaros, mostraram que o efeito residual foi mais importante no controle do que o efeito tópico. Entretanto, os dois efeitos somados melhoraram muito a eficiência dos produtos. A combinação desses efeitos geralmente ocorre em condições de campo. Aqueles ácaros que estavam abrigados e não receberam o produto diretamente sobre o idiossoma certamente entrarão posteriormente em contato com o resíduo do produto ao caminharem sobre a superfície tratada. Isso demonstra a importância de uma aplicação bem feita, atingindo toda a superfície foliar, ramos e frutos, não possibilitando locais de escape para a praga.

\section{Persistência}

Quanto à persistência dos produtos sobre as folhas, somente o fenbutatin oxide e o azocyclotin mostraram-se persistentes no controle de todas as fases pósembrionárias de $B$. phoenicis, ou seja, apresentaram mais de $80 \%$ de eficiência de controle além de 30 dias após a aplicação (DAA). O abamectin apresentou-se como persistente para o controle de larvas (> 30 DAA), moderadamente persistente para ninfas $(80 \%$ de eficiência entre 16 e 30 DAA) e levemente persistente para adultos de B. phoenicis $(80 \%$ de eficiência entre 6 e 15 DAA). A menor dosagem do emamectin apresentou-se levemente persistente para larvas (80\% de eficiência entre 6 e 15 DAA) e de baixa persistência para ninfas e adultos ( $80 \%$ de eficiência entre 0 e 5 DAA). Já a maior dosagem do emamectin foi levemente persistente para todas as fases pós-embrionárias de B. phoenicis $(80 \%$ de eficiência entre 5 e 15 DAA). O hexythiazox não apresentou nenhum efeito residual para nenhuma das fases pósembrionárias do ácaro (Tabelas 9). Para O. ilicis, o abamectin apresentou baixa persistência ( $>80 \%$ entre 0 e 5 DAA) para larvas, ninfas e adultos. Emamectin apresentou baixa persistência para larvas e nenhum efeito para ninfas e adultos, e fenbutatin oxide e azocyclotin apresentaram persistência no controle de todas as fases pós-embrionárias de O. ilicis (>80\% após 30

Ciênc. agrotec., Lavras, v. 28, n. 2, p. 269-281, mar./abr., 2004 
DAA). Hexythiazox não apresentou nenhum efeito de persistência no controle das fases pós-embrionárias do ácaro-vermelho do cafeeiro (Tabela 10).

\section{Seletividade}

Quanto ao efeito dos produtos sobre ácaros predadores, o abamectin apresentou-se como levemente e moderadamente nocivo (classes 2 e 3 ) e emamectin mostrou-se inócuo e levemente nocivo (classes 1 e 2) aos predadores
E. alatus e E. citrifolius, respectivamente. Emamectin causou baixa mortalidade e baixo efeito na reprodução dos fitoseídeos estudados (Tabelas 11 e 12). Fenbutatin oxide foi inócuo a E. citrifolius e levemente nocivo a E. alatus. Hexythiazox foi inócuo (classe 1), ao passo que o azocyclotin foi nocivo (classe 4) para as duas espécies de ácaros predadores estudadas (Tabelas $11 \mathrm{e}$ 12).

TABELA 7 - Número médio de larvas, ninfas e adultos de Brevipalpus phoenicis vivos 48 horas após a aplicação tópica mais efeito residual dos tratamentos e porcentagem de eficiência de controle (\% Efic.) $(\mathrm{n}=10)$.

\begin{tabular}{|c|c|c|c|c|c|c|c|}
\hline \multirow[b]{2}{*}{ Tratamentos } & \multirow{2}{*}{$\begin{array}{c}\text { Dosagem } \\
\text { por ha }\end{array}$} & \multicolumn{2}{|c|}{ Larvas } & \multicolumn{2}{|c|}{ Ninfas } & \multicolumn{2}{|c|}{ Adultos } \\
\hline & & Média $^{2}$ & $\begin{array}{c}\% \\
\text { Efic. }\end{array}$ & Média $^{2}$ & $\begin{array}{c}\% \\
\text { Efic. }\end{array}$ & Média $^{2}$ & $\begin{array}{c}\% \\
\text { Efic. }\end{array}$ \\
\hline Testemunha & - & $9,8 \mathrm{a}$ & - & $9,5 \mathrm{a}$ & - & $8,3 \mathrm{a}$ & - \\
\hline Abamectin (Vertimec $18 \mathrm{CE}$ ) & 300 & $0,0 \mathrm{c}$ & 100,0 & $0,0 \mathrm{c}$ & 100,0 & $0,0 \mathrm{~b}$ & 100,0 \\
\hline Emamectin (Proclaim 5 SG) & 200 & $0,3 \mathrm{~b}$ & 97,0 & $0,7 \mathrm{~b}$ & 92,6 & $0,0 \mathrm{~b}$ & 100,0 \\
\hline Emamectin (Proclaim 5 SG) & 400 & $0,0 \mathrm{c}$ & 100,0 & $0,0 \mathrm{c}$ & 100,0 & $0,0 \mathrm{~b}$ & 100,0 \\
\hline Fenbutatin oxide (Torque 500 SC) & 800 & $0,0 \mathrm{c}$ & 100,0 & $0,0 \mathrm{c}$ & 100,0 & $0,0 \mathrm{~b}$ & 100,0 \\
\hline Azocyclotin (Peropal 250 PM) & 1000 & $0,0 \mathrm{c}$ & 100,0 & $0,0 \mathrm{c}$ & 100,0 & $0,0 \mathrm{~b}$ & 100,0 \\
\hline Hexythiazox (Savey 500 PM) & 30 & $9,7 \mathrm{a}$ & 1,0 & $9,5 \mathrm{a}$ & 0,0 & $8,3 \mathrm{a}$ & 0,0 \\
\hline $\mathrm{CV}(\%)$ & & 7,2 & & 10,3 & & 11,0 & \\
\hline
\end{tabular}

g ou ml do produto comercial / 1000 litros de água.

${ }^{2}$ Médias seguidas de mesma letra na coluna não diferem entre si pelo teste de Duncan $(\mathbf{p}<0,05)$.

TABELA 8 - Número médio de larvas, ninfas e adultos de Oligonychus ilicis vivos 48 horas após a aplicação tópica mais efeito residual dos tratamentos e porcentagem de eficiência de controle (\% Efic.) $(n=10)$.

\begin{tabular}{|c|c|c|c|c|c|c|c|}
\hline \multirow[b]{2}{*}{ Tratamentos } & \multirow{2}{*}{$\begin{array}{c}\text { Dosagem } \\
\text { por ha }\end{array}$} & \multicolumn{2}{|c|}{ Larvas } & \multicolumn{2}{|c|}{ Ninfas } & \multicolumn{2}{|c|}{ Adultos } \\
\hline & & Média $^{2}$ & $\begin{array}{c}\% \\
\text { Efic. }\end{array}$ & Média' & $\begin{array}{c}\% \\
\text { Efic. }\end{array}$ & Média $^{2}$ & $\begin{array}{c}\% \\
\text { Efic. }\end{array}$ \\
\hline Testemunha & - & $9,8 \mathrm{a}$ & - & $9,8 \mathrm{a}$ & - & $8,7 \mathrm{a}$ & - \\
\hline Abamectin (Vertimec $18 \mathrm{CE}$ ) & 300 & $0,0 \quad \mathrm{e}$ & 100,0 & $0,0 \quad \mathrm{e}$ & 100,0 & $0,0 \quad \mathrm{~d}$ & 100,0 \\
\hline Emamectin (Proclaim 5 SG) & 200 & $4,7 \mathrm{~b}$ & 52,0 & $6,8 \mathrm{~b}$ & 30,6 & $6,3 \mathrm{~b}$ & 27,6 \\
\hline Emamectin (Proclaim 5 SG) & 400 & $3,2 \mathrm{c}$ & 67,3 & $4,2 \mathrm{c}$ & 57,1 & $4,0 \mathrm{c}$ & 54,0 \\
\hline Fenbutatin oxide (Torque 500 SC) & 800 & $1,2 \mathrm{~d}$ & 87,8 & $1,8 \quad \mathrm{~d}$ & 81,6 & $0,2 \quad \mathrm{~d}$ & 97,7 \\
\hline Azocyclotin (Peropal 250 PM) & 1000 & $0,0 \quad \mathrm{e}$ & 100,0 & $0,0 \quad \mathrm{e}$ & 100,0 & $0,0 \quad \mathrm{~d}$ & 100,0 \\
\hline Hexythiazox (Savey 500 PM) & 30 & $8,0 \mathrm{a}$ & 18,4 & $9,8 \mathrm{a}$ & 0,0 & $8,8 \mathrm{a}$ & 0,0 \\
\hline $\mathrm{CV}(\%)$ & & 15,6 & & 12,0 & & 8,8 & \\
\hline
\end{tabular}

g ou ml do produto comercial / 1000 litros de água.

${ }^{2}$ Médias seguidas de mesma letra na coluna não diferem entre si pelo teste de Duncan $(\mathbf{p}<0,05)$. 
TABELA 9 - Porcentagem de eficiência dos produtos no controle de larvas (L), ninfas (N) e adultos (A) de Brevipalpus phoenicis em função da persistência sobre as folhas de cafeeiro aos $0,5,15$ e 30 dias após a aplicação (DAA).

\begin{tabular}{|c|c|c|c|c|c|c|c|c|c|c|c|c|c|}
\hline \multirow{3}{*}{ Tratamentos $^{1}$} & \multirow{3}{*}{$\begin{array}{c}\text { Dosagem } \\
\text { g ou } \\
\text { ml/ha }\end{array}$} & \multicolumn{12}{|c|}{ Porcentagem de Eficiência / Fase Pós-embrionária / Dias Após a Aplicação } \\
\hline & & \multicolumn{3}{|c|}{ O DAA } & \multicolumn{3}{|c|}{5 DAA } & \multicolumn{3}{|c|}{15 DAA } & \multicolumn{3}{|c|}{30 DAA } \\
\hline & & $\mathbf{L}$ & $\mathbf{N}$ & $\mathbf{A}$ & $\mathbf{L}$ & $\mathbf{N}$ & $\mathbf{A}$ & $\mathbf{L}$ & $\mathbf{N}$ & $\mathbf{A}$ & $\mathrm{L}$ & $\mathrm{N}$ & A \\
\hline Hexythiazox & 30 & 0,0 & 50,0 & 23,0 & 0,0 & 5,3 & 0,0 & 5,7 & 1,1 & 0,0 & 4,4 & 22,7 & 6,5 \\
\hline Emamectin & 200 & 100,0 & 100,0 & 100,0 & 100,0 & 50,5 & 67,6 & 19,5 & 5,7 & 0,0 & 27,2 & 29,9 & 16,9 \\
\hline Abamectin & 300 & 100,0 & 100,0 & 100,0 & 96,5 & 98,0 & 100,0 & 96,6 & 86,2 & 71,3 & 91,3 & 67,0 & 52,0 \\
\hline Emamectin & 400 & 100,0 & 100,0 & 100,0 & 96,5 & 100,0 & 100,0 & 19,5 & 37,6 & 24,1 & 26,1 & 5,1 & 18,2 \\
\hline Fenbutatin oxide & 800 & 100,0 & 100,0 & 100,0 & 100,0 & 100,0 & 100,0 & 85,1 & 100,0 & 100,0 & 100,0 & 100,0 & 100,0 \\
\hline Azocyclotin & 1000 & 100,0 & 100,0 & 100,0 & 100,0 & 100,0 & 100,0 & 100,0 & 100,0 & 100,0 & 100,0 & 100,0 & 100,0 \\
\hline
\end{tabular}

${ }^{1}$ Produtos comerciais: Abamectin (Vertimec $18 \mathrm{CE}$ ), emamectin (Proclaim 5 SG, fenbutatin oxide (Torque 500 SC), azocyclotin (Peropal 250 PM) e hexythiazox (Savey 500 PM).

TABELA 10 - Porcentagem de eficiência dos produtos no controle de larvas (L), ninfas (N) e adultos (A) de Oligonychus ilicis em função da persistência sobre as folhas de cafeeiro aos $0,5,15$ e 30 dias após a aplicação (DAA).

\begin{tabular}{|c|c|c|c|c|c|c|c|c|c|c|c|c|c|}
\hline \multirow{3}{*}{ Tratamentos $^{1}$} & \multirow{3}{*}{$\begin{array}{c}\text { Dosagem } \\
\text { g ou } \\
\text { ml/ha }\end{array}$} & \multicolumn{12}{|c|}{ Porcentagem de Eficiência / Fase Pós-embrionária / Dias Após a Aplicação } \\
\hline & & \multicolumn{3}{|c|}{ O DAA } & \multicolumn{3}{|c|}{5 DAA } & \multicolumn{3}{|c|}{15 DAA } & \multicolumn{3}{|c|}{30 DAA } \\
\hline & & $\mathbf{L}$ & $\mathbf{N}$ & A & $\mathbf{L}$ & $\mathbf{N}$ & A & $\mathbf{L}$ & $\mathbf{N}$ & $\mathbf{A}$ & $\mathbf{L}$ & $\mathbf{N}$ & $\mathbf{A}$ \\
\hline Hexythiazox & 30 & 2,0 & 0,0 & 3,1 & 0,0 & 17,0 & 15,2 & 0,0 & 0,0 & 17,2 & 0,0 & 2,1 & 7,2 \\
\hline Emamectin & 200 & 47,0 & 26,5 & 11,2 & 0,0 & 0,0 & 9,8 & 12,4 & 0,0 & 5,4 & 7,6 & 8,4 & 12,4 \\
\hline Emamectin & 400 & 88,0 & 57,0 & 31,6 & 8,6 & 17,0 & 0,0 & 19,6 & 0,0 & 0,0 & 15,2 & 8,4 & 5,2 \\
\hline Abamectin & 300 & 100,0 & 100,0 & 100,0 & 19,4 & 30,0 & 21,7 & 17,5 & 0,0 & 0,0 & 5,4 & 10,5 & 0,0 \\
\hline Fenbutatin oxide & 800 & 100,0 & 87,8 & 92,9 & 100,0 & 100,0 & 100,0 & 100,0 & 86,0 & 96,8 & 87,0 & 82,1 & 91,8 \\
\hline Azocyclotin & 1000 & 100,0 & 100,0 & 100,0 & 100,0 & 100,0 & 100,0 & 100,0 & 100,0 & 100,0 & 100,0 & 100,0 & 100,0 \\
\hline
\end{tabular}

${ }^{1}$ Produtos comerciais: Abamectin (Vertimec $18 \mathrm{CE}$ ), emamectin (Proclaim 5 SG, fenbutatin oxide (Torque 500 SC), azocyclotin (Peropal 250 PM) e hexythiazox (Savey 500 PM). 
TABELA 11 - Toxicidade de agroquímicos a Euseius citrifolius em teste residual de laboratório a $25 \pm 2^{\circ} \mathrm{C}, 70 \pm 10 \%$ de UR e 14 horas de fotofase (resíduo de $1,68 \pm 0,36 \mathrm{mg} / \mathrm{cm}^{2} \mathrm{em}$ superfície de vidro).

\begin{tabular}{lcccccc}
\hline \multicolumn{1}{c}{ Nome técnico } & $\begin{array}{c}\text { Dosagem } \\
\text { por ha }\end{array}$ & $\mathbf{M}_{\mathbf{c}}{ }^{\mathbf{2}}(\boldsymbol{\%})$ & $\mathbf{R}^{\mathbf{3}}$ & $\mathbf{E}_{\mathbf{r}}{ }^{\mathbf{4}}$ & $\mathbf{E}^{\mathbf{5}}(\boldsymbol{\%})$ & $\mathbf{C l a s s e}^{\mathbf{6}}$ \\
\hline Emamectin (Proclaim 5 SG) & 200 & $-7,14$ & 8,23 & 1,11 & $-18,75$ & 1 \\
Fenbutatin oxide (Torque 500 SC) & 800 & 67,86 & 9,67 & 2,44 & 21,62 & 1 \\
Hexythiazox (Savey 500 PM) & 30 & 0,00 & 10,57 & 0,94 & 6,33 & 1 \\
Abamectin (Vertimec 18 CE) & 300 & 34,61 & 3,53 & 0,95 & 38,14 & 2 \\
Emamectin (Proclaim 5 SG) & 400 & 10,00 & 5,18 & 0,54 & 51,56 & 2 \\
Azocyclotin (Peropal 250 PM) & 1000 & 100,00 & 0,00 & 0,00 & 100,00 & 4 \\
\hline
\end{tabular}

${ }^{1} \mathrm{~g}$ ou $\mathbf{m l}$ do produto comercial/1000 litros de água.

${ }^{2} M_{c}=$ Mortalidade corrigida (\%). $M_{c}=$ (ácaros vivos test. - ácaros vivos trat.)/ácaros vivos test. $\mathbf{x} 100$.

${ }^{3} R$ = Produção média de ovos/fêmeas. $R=n^{0}$ ovos viáveis/ $n^{0}$ de fêmeas.

${ }^{4}$ Efeito na reprodução. $\mathbf{E r}=\mathbf{R}_{\text {trat }} / \mathbf{R}_{\text {test }}$.

${ }^{5}$ Efeito total ou adverso. $E=100 \%$ - $(100 \%$ - Mc $) \times$ Er.

${ }^{6}$ Classes toxicidade segundo a IOBC/WPRS: Classe $1=\mathrm{E}<30 \%$ (não nocivo); Classe 2 = $30<\mathrm{E}<80$ (levemente nocivo); Classe $3=80<E<99$ (moderadamente nocivo); Classe $4=\mathrm{E}>99 \%$ (nocivo).

TABELA 12 - Toxicidade de agroquímicos a Euseius alatus em teste residual de laboratório a $25 \pm 2^{\circ} \mathrm{C}, 70 \pm 10 \%$ de UR e 14 horas de fotofase (resíduo de $1,68 \pm 0,36 \mathrm{mg} / \mathrm{cm}^{2}$ em superfície de vidro).

\begin{tabular}{lcccccc}
\hline \multicolumn{1}{c}{ Nome técnico } & $\begin{array}{c}\text { Dosagem } \\
\text { por ha }^{\mathbf{1}}\end{array}$ & $\mathbf{M}_{\mathbf{c}}{ }^{\mathbf{2}}(\boldsymbol{\%})$ & $\mathbf{R}^{\mathbf{3}}$ & $\mathbf{E}_{\mathbf{r}}{ }^{\mathbf{4}}$ & $\mathbf{E}^{\mathbf{5}}(\boldsymbol{\%})$ & Classe $^{\mathbf{6}}$ \\
\hline Emamectin (Proclaim 5 SG) & 400 & $-4,17$ & 4,60 & 0,87 & 9,45 & 1 \\
Hexythiazox (Savey 500 PM) & 30 & $-12,00$ & 7,36 & 1,09 & $-22,62$ & 1 \\
Emamectin (Proclaim 5 SG) & 200 & 17,39 & 4,79 & 0,65 & 46,47 & 2 \\
Fenbutatin oxide (Torque 500 SC) & 800 & 36,00 & 5,19 & 0,75 & 52,02 & 2 \\
Abamectin (Vertimec 18 CE) & 300 & 50,00 & 1,40 & 0,20 & 89,90 & 3 \\
Azocyclotin (Peropal 250 PM) & 1000 & 100,00 & 0,00 & 0,00 & 100,00 & 4 \\
\hline
\end{tabular}

${ }^{1} \mathrm{~g}$ ou $\mathbf{~ m l ~ d o ~ p r o d u t o ~ c o m e r c i a l / 1 0 0 0 ~ l i t r o s ~ d e ~ a ́ g u a . ~}$

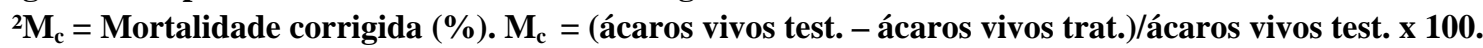

${ }^{3} R=$ Produção média de ovos/fêmeas. $R=n^{0}$ ovos viáveis/ $n^{0}$ de fêmeas.

${ }^{4}$ Efeito na reprodução. $\mathbf{E r}=\mathbf{R}_{\text {trat }} / \mathbf{R}_{\text {test }}$.

${ }^{5}$ Efeito total ou adverso. $\mathrm{E}=\mathbf{1 0 0 \%}-(\mathbf{1 0 0 \%}-\mathrm{Mc}) \mathrm{x}$ Er.

${ }^{6}$ Classes toxicidade segundo a IOBC/WPRS: Classe $1=\mathrm{E}<30 \%$ (não nocivo); Classe $2=30<\mathrm{E}<80$ (levemente nocivo); Classe $3=80<\mathrm{E}<99$ (moderadamente nocivo); Classe $4=\mathrm{E}>99 \%$ (nocivo).

\section{CONCLUSÕES}

a) Abamectin (Vertimec $18 \mathrm{CE}$ ) e emamectin (Proclaim 5 SG) apresentam baixo efeito ovicida aos ácaros B. phoenicis e O. ilicis. b) Abamectin e emamectin apresentam eficiente efeito residual e tópico no controle das fases pósembrionárias do ácaro da mancha-anular do cafeeiro $(B$. phoenicis) e a combinação dos dois efeitos melhora ainda mais a eficiência dos produtos. 
c) Abamectin apresenta eficiente efeito residual e tópico no controle das fases pós-embrionárias do ácarovermelho do cafeeiro (O. ilicis) e a combinação dos dois efeitos melhora ainda mais a eficiência do produto.

d) Mesmo sob drásticas condições em laboratório, o abamectin e o emamectin apresentam seletividade fisiológica aos fitoseídeos E. alatus e E. citrifolius.

e) Considerando a eficiência do abamectin e do emamectin no controle de B. phoenicis, e seletividade para fitoseídeos, conclui-se que esses produtos podem ser utilizados em programas de manejo integrado do ácaro em cafeeiro. A mesma consideração pode ser feita para a utilização do abamectin no manejo integrado de $O$. ilicis.

\section{AGRADECIMENTOS}

À Syngenta Proteção de Cultivos Ltda., pelo apoio à nossa participação no Syngenta Entomological Advisory Group - SEAG 2002, e ao CNPq e Consórcio Brasileiro de Pesquisa e Desenvolvimento do Café CBP\&D/Café, pelas bolsas concedidas.

\section{REFERÊNCIAS BIBLIOGRÁFICAS}

ABBOTT, W. S. A method of computing the effectiveness of an insecticide. Journal of Economic Entomology, Lanham, v. 18, p. 265-267, 1925.

AMARAL, J. F. O ácaro dos cafezais. Boletim da Superintendência dos Serviços do Café, São Paulo, v. 26, n. 296, p. 846-848, 1951.

A INFESTAÇÃO de ácaros nos cafezais. O Biológico, São Paulo, v. 17, n. 7, p. 130, 1951.

BAKKER, F. M.; GROVE, A.; BLÜMEL, S.; CALIS, J.; OOMEN, P. Side-effect test for phytoseiids and their rearing methods. IOBC/WPRS Bulletin, Montfavet, v. 15, n. 3, p. 61-81, 1992.

BEVENGA, S. R.; GRAVENA, S.; CAETANO, A. C.; SILVA, J. L.; LINARDI, M. S.; ARAÚJO JÚNIOR, N. Manejo de Leucoptera coffeella, Brevipalpus phoenicis e Oligonychus ilicis com abamectin e emamectin e sua influência sobre inimigos naturais em cafeeiro. In: CONGRESSO BRASILEIRO DE ENTOMOLOGIA, 19., 2002, Manaus. Resumos... Manaus: Sociedade Entomológica do Brasil, 2002. p. 272, 328 p.

CALZA, R.; SAUER, H. F. G. A aranha vermelha dos cafezais. O Biológico, São Paulo, v. 18, n. 12, p. 201208, 1952.
CHAGAS, C. M. Associação do ácaro Brevipalpus phoenicis (Geijskes) à mancha anular do cafeeiro. $\mathbf{O}$ Biológico, São Paulo, v. 39, n. 9, p. 229-232, 1973.

CHAGAS, C. M. Viroses, ou doenças semelhantes transmitidas por ácaros tenuipalpídeos: mancha anular do cafeeiro e leprose dos citros. Fitopatologia Brasileira, São Paulo, v. 13, n. 2, p. 92, jul. 1988.

CHIAVEGATO, L. G. Ácaros da cultura dos citros. In: RODRIGUEZ, O.; VIÉGAS, F.; POMPEU JÚNIOR, J.; AMARO, A. A. (Eds.). Citricultura brasileira. 2. ed. Campinas: Fundação Cargill, 1991. v. 2, p. 601-641, $941 \mathrm{p}$.

CHIAVEGATO, L. G. Biologia do ácaro Brevipalpus phoenicis em citros. Pesquisa Agropecuária Brasileira, Brasília, v. 21, n. 8, p. 813-816, ago. 1986.

CHIAVEGATO, L. G.; KHARFAN, P. R. Comportamento do ácaro da leprose Brevipalpus phoenicis (G.) (Acari: Tenuipalpidae) em citros. Anais da Sociedade Entomológica do Brasil, Itabuna, v. 22, n. 2, p. 355359, set. 1993.

CHIAVEGATO, L. G.; MISCHAN, M. M.; SILVA, M. A. Prejuízos e transmissibilidade de sintomas de leprose pelo ácaro Brevipalpus phoenicis (Geijskes, 1939) Sayed, 1946 (Acari, Tenuipalpidae) em citros. Científica, São Paulo, v. 10, n. 2, p. 265-271, 1982.

FIGUEIRA, A. R.; REIS, P. R.; CARVALHO, V. L.; PINTO, C. S. Coffee ringspot virus is becoming a real problem to Brazilian coffee growers. In: INTERNATIONAL CONGRESS OF VIROLOGY, 10., 1996, Jerusalem, Israel. Abstracts... Jerusalem: [s.n.], 1996. p. 203.

HASSAN, S. A.; BIGLER, F.; BOGENSCHÜTZ, H.; BOLLER, E.; BRUN, J.; CALIS, J. N. M.; COREMANSPELSENEER, J.; DUSO, C.; GROVE, A.; HEIMBACH, U.; HELYER, N.; HOKKANEN, H.; LEWIS, G. B.; MANSOUR, F.; MORETH, L.; POLGAR, L.; SAMSOEPETERSEN, L.; SAUPHANOR, B.; STÄUBLI, A.; STERK, G.; VAINIO, A.; VEIRE, M. van de; VIGGIANI, G.; VOGT, H. Results of the sixth joint pesticide testing programme of the IOBC/WPRS: workig group "Pesticides and Beneficial Organisms". Entomophaga, Paris, v. 39, n. 1, p. 107-119, 1994.

INSTITUTO BRASILEIRO DO CAFÉ. Cultivo do café conilon. In: _. Cultura do café no Brasil: manual de recomendações. Rio de Janeiro: IBC/GERCA, 1985. p. $527-556,580 \mathrm{p}$. 
MATIELLO, J. B. Novas condições de ocorrência de mancha anular do cafeeiro. In: CONGRESSO BRASILEIRO DE PESQUISAS CAFEEIRAS, 14., 1987, Campinas. Resumos... Rio de Janeiro: MIC /IBC, 1987. p. 6, 323 p.

McMURTRY, J. A.; CROFT, B. A. Life-styles of phytoseiid mites and their roles in biological control. Annual Review of Entomology, Palo Alto, v. 42, p. 291-321, 1997.

OLIVEIRA, C. A. L.; REIFF, E. T. Influência do volume de calda aplicada de acaricidas no controle do Brevipalpus phoenicis, transmissor da mancha anular do cafeeiro. In: CONGRESSO BRASILEIRO DE PESQUISAS CAFEEIRAS, 24., 1998, Poços de Caldas. Trabalhos Apresentados... Rio de Janeiro: MAA/SDR/PROCAFÉ/PNFC, 1998. p. 140, 319 p.

OVERMEER, W. P. J.; ZON, A. Q. van. A standardized method for testing the side effects of pesticides on the predaceous mite, Amblyseius potentillae (Acarina: Phytoseiidae). Entomophaga, Paris, v. 27, n. 4, p. $357-$ 64, 1982.

PALLINI FILHO, A.; MORAES, G. J.; BUENO, V. H. P. Ácaros associados ao cafeeiro (Coffea arabica L.) no Sul de Minas Gerais. Ciência e Prática, Lavras, v. 16, n. 3, p. 303-307, jul./set. 1992.

PAPA, G. Ocorrência, sintomas e controle do ácaro-daleprose, Brevipalpus phoenicis, (Geijskes, 1939) (Acari: Tenuipalpidae), na cultura do café. In: CONGRESSO BRASILEIRO DE PESQUISAS CAFEEIRAS, 23., 1997, Manhuaçu. Trabalhos Apresentados... Rio de Janeiro: MAA/SDR/PROCAFÉ/ PNFC, 1997. p. 231233, $249 \mathrm{p}$.

REIS, P. R. Ácaros de algumas fruteiras de clima tropical e subtropical e seus hospedeiros. Lavras: ESAL, 1974. 32 p. (Boletim Técnico, Série Pesquisa, 3).

REIS, P. R.; ALVES, E. B. Criação do ácaro predador Iphiseiodes zuluagai Denmark \& Muma (Acari: Phytoseiidae) em laboratório. Anais da Sociedade Entomológica do Brasil, Itabuna, v. 26, n. 3, p. 565-568, dez. 1997.
REIS, P. R.; ALVES, E. B.; SOUSA, E. O. Biologia do ácaro-vermelho do cafeeiro Oligonychus ilicis (McGregor, 1917). Ciência e Agrotecnologia, Lavras, v. 21, n. 3, p. 260-266, jul./set. 1997.

REIS, P. R.; CHAGAS, S. J. R. Relação entre o ataque do ácaro-plano e da mancha-anular com indicadores da qualidade do café. Ciência e Agrotecnologia, Lavras, v. 25 , n. 1, p. 72-76, jan./fev. 2001.

REIS, P. R.; CHIAVEGATO, L. G.; MORAES, G. J.; ALVES, E. B.; SOUSA, E. O. Seletividade de agroquímicos ao ácaro predador Iphiseiodes zuluagai Denmark \& Muma (Acari: Phytoseiidae). Anais da Sociedade Entomológica do Brasil, Itabuna, v. 27, n. 2, p. 265-274, jun. 1998.

REIS, P. R.; SOUZA, J. C. Pragas do cafeeiro. In: RENA, A. B.; MALAVOLTA, E.; ROCHA, M.; YAMADA, T. (Eds.). Cultura do cafeeiro: fatores que afetam a produtividade. Piracicaba: POTAFOS, 1986. p. $338-378,447$ p.

REIS, P. R.; SOUSA, E. O.; ALVES, E. B. Seletividade de produtos fitossanitários ao ácaro predador Euseius alatus DeLeon (Acari: Phytoseiidae). Revista Brasileira de Fruticultura, Jaboticabal, v. 21, n. 3, p. 350-355, dez. 1999.

REIS, P. R.; SOUZA, J. C.; PEDRO NETO, M.; TEODORO, A. V. Flutuação populacional do ácaro da mancha-anular do cafeeiro e seus inimigos naturais. In: SIMPÓSIO DE PESQUISA DOS CAFÉS DO BRASIL, 2000, Poços de Caldas. Resumos Expandidos... Brasília: EMBRAPA-CAFÉ, 2000. v. 2, p. 1210-1212, $1490 \mathrm{p}$.

REIS, P. R.; SOUZA, J. C.; SOUSA, E. O.; TEODORO, A. V. Controle do Brevipalpus phoenicis em cafeeiro com produtos seletivos a ácaros predadores. Manejo Integrado de Plagas y Agroecologia, Costa Rica, v. 64, p. 55-61, jun. 2002.

TRINDADE, M. L. B.; CHIAVEGATO, L. G. Caracterização biológica dos ácaros Brevipalpus obovatus D., B. californicus B. e B. phoenicis G. (Acari: Tenuipalpidae). Anais da Sociedade Entomológica do Brasil, Itabuna, v. 23, n. 2, p. 189-195, ago. 1994. 